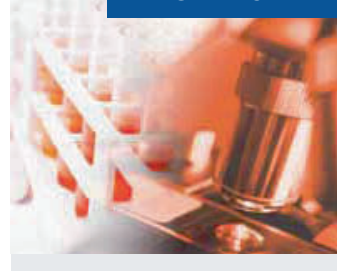

Berichte aus der Pharmaindustrie

\title{
NPH-Insuline
}

\section{Cave Dosierfehler bei schlechtem Durchmischen}

- Vor der Applikation von NPH-Insulinen muss der Patient die Ampulle bis zu 20mal schwenken, um die Insulinuspension homogen zu durchmischen. Viele Patienten kürzen den Vorgang im Alltag ab und schütteln die Ampulle nur ein paar Mal. Sie riskieren dabei bei den meisten NPH-Insulinen Dosierungsfehler, was zu Hypo- und Hyperglykämien führen kann. Einzig das NPH-Insulin Insuman Basal ${ }^{\circledR}$ verzeiht Durchmischungsfehler und bietet eine ausreichende Dosiergenauigkeit bei nur drei Mischvorgängen, vermutlich weil es als einziges Insulinpräparat drei Mischkugeln aus Metall in der Kartusche hat.

Dies folgt aus einer Studie, bei der die Dosiergenauigkeit von fünf NPH-Insulinpens nach 3, 6, 10 und 20 Mischvorgängen verglichen wurde. Bei vorschriftsmäßigen 20 Mischvorgängen boten alle Präparate eine hohe Dosiergenauigkeit. Bei nur 3-6
Mischvorgängen zeigen außer dem Tactipen alle NPH-Insuline Unter- und Überdosierungen von $5 \%-35 \%$. Wer schlecht schüttelt, ist mit dem NPHInsulin von Sanofi-Aventis auf der sicheren Seite.

Das Unternehmen hat zudem sein Insulin-PenAngebot um den wiederverwendbaren Clickstar $^{\circledR}$ erweitert, der in den drei Farben schwarz, silber und grau für alle Sanofi-Insuline (Lantus ${ }^{\circledR}$, Apidra ${ }^{\circledR}$, Insuman ${ }^{\circledR}$ ) angeboten wird. Bei der Entwicklung des strapazierfähigen (4 Jahre Garantie), 100\% mechanischen Pens wurde v.a. auf hohe Ergometrie Wert gelegt: Der Pen ist sehr einfach zu bedienen. Die Injektionshilfe wurde mit dem Good Design Award vom Chicago Athenaeum Museum of Architecture and Design ausgezeichnet. red

Pressekonferenz bei der DDG-Jahrestagung 13.5.2010 in Stuttgart, Veranstalter: Sanofi-Aventis

\section{Auch über zwei Jahre sicher und effektiv bei Typ-2-Diabetes Bei Sitagliptin stimmt das Gesamtpaket der Eigenschaften}

- Was letztlich zählt, ist das Gesamtpaket der Eigenschaften eines Antidiabetikums. Der DPP-4-Hemmer Sitagliptin schneidet hier bei Wirksamkeit und Verträglichkeit im Vergleich zu einem Sulfonylharnstoff auch längerfristig gut ab. Das hat eine 2-JahresStudie mit einer Post-hoc-Analyse ergeben.

Die Studie mit 1172 Typ-2-Diabetikern war auf zwei Jahre angelegt und dafür konzipiert, anhand des $\mathrm{HbA}_{1 \mathrm{c}}$ die Langzeiteffizienz von Sitagliptin (z.B. Januvia $^{\circledR}$, plus Metformin Janumet $\left.{ }^{\circledR}\right)$ zu prüfen [1]. Dafür erhielten die mit $\geq 1500 \mathrm{mg} / \mathrm{d}$ Metformin unzureichend eingestellten Patienten randomisiert zusätzlich $100 \mathrm{mg} / \mathrm{d}$ Sitagliptin oder den Sulfonylharnstoff (SU) Glipizid (5-20 mg/d). Nach 104 Wochen war die $\mathrm{HbA}_{1 \mathrm{c}}$-Senkung (Basis im Mittel 7,5\%) mit beiden Präparaten ähnlich (Gliptin -0,54 vs. SU -0,51\%Punkte). Nachdem der $\mathrm{HbA}_{1 \mathrm{c}}$ nach 24 Wochen seinen Tiefpunkt erreicht hatte, stieg er zwar im Rahmen der Diabetesprogression wieder etwas an, mit dem Gliptin jedoch weniger (16\% vs. $21 \% / \mathrm{Jahr})$. Von Hypoglykämien mit Sitagliptin berichteten $5 \%$, mit dem SU 33\% der Patienten. Auch die Gewichtsentwicklung mit dem Gliptin war vorteilhaft $(-1,6 \mathrm{~kg}$ vs. $+0,7 \mathrm{~kg}$ mit

Abb. 1: Mit Sitagliptin erreichten 31,7\% bzw. $\mathbf{5 5 , 8} \%$ der Patienten den kombinierten Endpunkt. zudem eine stabile Betazellfunktion mit Sitagliptin, die mit dem SU nachgelassen hatte.

Die 1-Jahres-Daten dieser Studie wurden dann post hoc separat analysiert [2]. Auch zu diesem Zeitpunkt war die Wirksamkeit des Gliptins der des SU ebenbürtig. Nun betrachteten die Forscher den kombinierten Endpunkt aus effektiver $\mathrm{HbA}_{1 \mathrm{c}}$-Senkung $>0,5 \%$-Punkte, Ausbleiben einer Gewichtszunahme und Ausbleiben von Hypoglykämien. Das Rennen ging deutlich zugunsten von Sitagliptin aus: Mit 38,1\% der 388 Patienten erreichten signifikant mehr diesen Endpunkt als mit Glipizid (11,8\% von 415 Patienten). Auch bei der seperaten Analyse der Patienten mit einem Ausgangs- $\mathrm{HbA}_{1 \mathrm{c}}<8 \%$ und $\geq 8 \%$ erreichten mit Sitagliptin signifikant mehr diesen Endpunkt (Abb.1). Prof. Michael Nauck, Bad Lauterberg, betonte auch anhand dieser Studie, dass SU ein nicht zu unterschätzendes Gefahrenpotenzial haben. Dies äußere sich bekanntlich in vermehrten Hypoglykämien. Zudem gebe es auch Hinweise, dass SU die kardiale Funktion beeinträchtigen und so kardiovaskuläre Ereignisse begünstigen könnten. Für DPP-4-Hemmer sei ein entgegengesetzter Effekt erkennbar. Nauck: „Warum also sollte ich Präparate mit Nachteilen wie Sulfonylharnstoffe verordnen, wenn es eine auch langfristig gleich wirksame, aber weniger riskante Alternative gibt?"

spa

1. Seck T, Nauck M, Sheng D et al. Int J Clin Pract 2010;64(5):562-76. 2. Seck T, Engel S, Willams-H DE et al. 580-p, Diabetes 2010;(59)A158.

Poster und Symposium bei der ADA-Jahrestagung, 28.6.2010 in Orlando/USA 